\title{
Polychronization: Computation With Spikes
}

\author{
Eugene M. Izhikevich *
}

January 26, 2005

\begin{abstract}
We present a minimal spiking network that can polychronize, i.e., exhibit persistent time-locked but not synchronous firing patterns with millisecond precision, as in synfire braids. The network consists of cortical spiking neurons with axonal conduction delays and spike-timingdependent plasticity (STDP); a ready-to-use MATLAB code is included. It exhibits sleep oscillations, gamma $(40 \mathrm{~Hz})$ rhythms, and other interesting regimes. Due to the interplay between the delays and STDP, the spiking neurons spontaneously self-organize into groups and generate polychronous persistent activity. The number of co-existing polychronous groups far exceeds the number of neurons in the network, resulting in an unprecedented memory capacity of the system.
\end{abstract}

\section{Introduction}

The artificial neural networks community has recently been invigorated by the idea, borrowed from studies of biological neural networks, that spike-timing could be used as an additional variable in the information processing by the brain. See, e.g., special issue of IEEE TNN on pulse-coupled neural networks (May 1999), special issue of Neuron on binding problem (September 1999), special issue of Neural Networks on spiking neurons (July 2001), and special issue of IEEE TNN on temporal coding (July 2004).

Spikes. When considering spiking neurons, most researchers emphasize synchrony of firing. Indeed, they reckon that if two or more neurons have a common postsynaptic target and fire synchronously, then their spikes arrive to the target at the same time, thereby evoking potent postsynaptic responses. If the neurons fire asynchronously, their spikes arrive to the postsynaptic target at different times evoking only weak or no response. An implicit assumption here is that the axonal conduction delays are negligible or equal.

${ }^{*}$ The Neurosciences Institute, 10640 John Jay Hopkins Drive, San Diego, CA, 92121, http://www.izhikevich.com, Eugene.Izhikevich@nsi.edu 
A

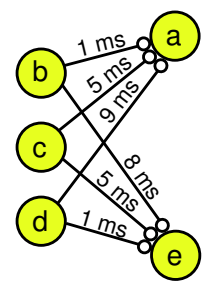

C

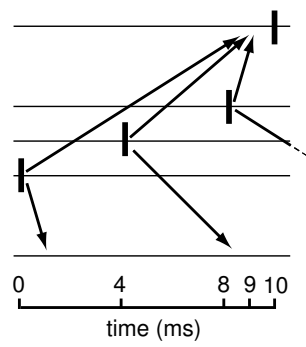

B

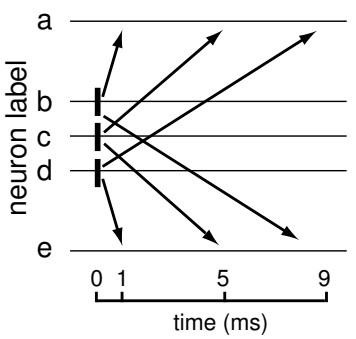

D

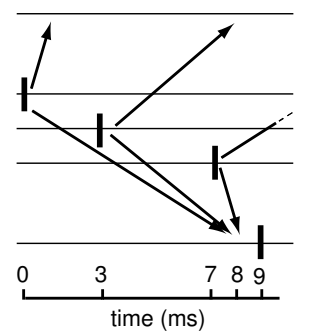

Figure 1: (A) Synaptic connections from neurons $b, c$, and $d$ to neurons $a$ and $e$ have different axonal conduction delays. (B, C, D) Firings of neurons are denoted by the vertical bars. Each arrow points to the spike arrival time to the postsynaptic neuron. (B) Synchronous firing is not effective to elicit a potent postsynaptic response since the spikes arrive to the postsynaptic neurons at different times. (C) The spiking pattern with neuron $d$ firing at $0 \mathrm{~ms}$, neuron $c$ firing at $4 \mathrm{~ms}$ and neuron $b$ firing at $8 \mathrm{~ms}$ is optimal to excite neuron $a$ because the spikes arrive at $a$ simultaneously. (D) The reverse order of firing is optimal to excite neuron $e$.

Delays A careful measurement of axonal conduction delays in the mammalian neocortex (Swadlow 1985, 1988, 1992) showed that they could be as small as 0.1 $\mathrm{ms}$ and as large as $40 \mathrm{~ms}$, depending on the type and location of the neurons. The majority of computational neuroscientists discard this finding as a nuisance that only complicates modeling. From a mathematical point of view, a system with delays is not finite- but infinite-dimensional, which indeed poses some mathematical and simulation difficulties.

In this paper we argue that an infinite dimensionality of spiking networks with axonal delays is not a nuisance, but an immense advantage that results in an unprecedented, possibly unlimited information capacity. In particular, there are stable firing patterns that are not possible without the delays.

Polychronization. To illustrate the main idea, consider neuron $a$ in Fig. 1A receiving inputs from neurons $b, c$, and $d$ with different conduction delays. 
Synchronous firing, as in Fig. 1B, is not effective to excite $a$, because the spikes arrive to $a$ at different times. To maximize the postsynaptic response in $a$, the presynaptic neurons should fire with the temporal pattern determined by the delays and depicted in Fig. 1C so that the spikes arrive at $a$ simultaneously. A different spike-timing pattern, as in Fig. 1D, excites neuron $e$.

We see that depending on the order and the precise timing of firing, the same three neurons can evoke a spike either in neuron $a$ or in neuron $e$, or possibly in some other neuron not shown in the figure. Notice how the conduction delays make this possible.

If $b, c$ and $d$ are sensory neurons driven by an external input, then the simple circuit in Fig. 1 can recognize and classify simple spatio-temporal patterns (Hopfield 1995, Seth et al. 2004). Indeed, sensory input as in Fig. 1C results in $(d, c, b, a)$ firing as a group with spike-timing pattern $(0,4,8,10)$ ms. Sensory input as in Fig. 1D results in a different set of neurons, namely $(b, c, d, e)$, firing as a group with a different spike-timing pattern, namely $(0,3,7,9)$ ms. Because the firings of these neurons are not synchronous, but time-locked to each other, we refer to such groups as polychronous, where poly means many and chronous means time or clock in Greek.

Networks. To explore the issue of spike-timing in networks with conduction delays, we simulated an anatomically realistic model consisting of 100,000 cortical spiking neurons having receptors with AMPA, NMDA, GABA $A_{A}$, and $\mathrm{GABA}_{\mathrm{B}}$ kinetics, and long-term and short-term synaptic plasticity (Izhikevich et al. 2004). We found that the network contains large polychronous groups, illustrated in Fig. 10, capable of recognizing and classifying quite complicated spatio-temporal patterns.

The existence of such groups was predicted by Bienenstock (1995, Appendix C) and Gerstner et al. (1996). However, no one has expected that the number of co-existing polychronous groups could be greater than the number of neurons in the network, sometimes even greater than the number of synapses. That is, each neuron was part of many groups, firing with one group at one time and with another group at another time. Retrospectively thinking, this is not surprising, since the networks we consider have delays, and hence are infinite-dimensional.

Novel Model of Computation. We view polychronous groups as representations of memorized stimuli. That is, every time a previously memorized stimulus is presented to the network, polychronous groups corresponding to this stimulus activate, i.e., the neurons comprising the groups fire spatio-temporal spiking pattern with a millisecond precision.

We find that there are usually more than one group corresponding to the same stimulus. Such a degeneracy (Edelman and Gally 2001) may be advantageous because of unreliable synaptic transmission, spiking noise, and synaptic turnover (Trachtenberg et al. 2002, Grutzendler et al. 2002). Since the number of groups far exceeds the number of neurons, or even the number of synapses, the network has an enormous memory capacity. Of course, we need to learn how 
to utilize this extraordinary property.

In this paper we present a minimal model that captures the essence of this phenomenon. The MATLAB code of the model, spnet, and its technical description is given in the Appendix. In Sect 2 we demonstrate that despite its simplicity, the model exhibits cortical-like dynamics, including sleep oscillations in the delta $(4 \mathrm{~Hz})$ frequency range, $40 \mathrm{~Hz}$ gamma oscillations, balance of excitation and inhibition, etc. In Sect. 3 we describe polychronous groups in detail. Our definition differs from the one used by Izhikevich et al. (2004), who relied on the existence of so-called anchor neurons and hence could not have more groups than neurons. In Sect. 4 we illustrate how polychronous groups contribute to cognitive information processing going beyond the Hopfield-Grossberg or liquidstate-machine paradigms. In Sect. 5 we discuss some open problems.

\section{Dynamics}

The model neural network, described in Appendix, preserves important ratios found in the mammalian cortex (Braitenberg and Schuz 1991). It consists of 1000 randomly connected excitatory (80\%) and inhibitory (20\%) neurons. The network is sparse with the probability of connection between any two neurons around 0.1. Behavior of each neuron is described by the simple spiking model (Izhikevich 2003), which can reproduce 20 most fundamental neurocomputational features of biological neurons summarized in Fig. 2. Despite its versatility, the model can be implemented efficiently (Izhikevich 2004). Since we cannot simulate an infinite-dimensional system on a finite-dimensional digital computer, we substitute the network by its finite-dimensional approximation having time resolution $1 \mathrm{~ms}$.

Plasticity. Synaptic connections among neurons have fixed conduction delays between 1 and $20 \mathrm{~ms}$. Synaptic weights evolve according to the spike-timingdependent plasticity (STDP) illustrated in Fig. 3 (Song et al. 2000). The magnitude of change of synaptic weight between a pre- and a post-synaptic neuron depends on the timing of spikes: If the pre-synaptic spike arrives to the post-synaptic neuron before the postsynaptic neuron fires, e.g., it causes the firing, the synapse is potentiated. Its weight is increased according to the positive part of the STDP curve in Fig. 3. If the pre-synaptic spike arrives to the postsynaptic neuron after it fired, i.e., it brings the late news, the synapse is depressed. Its weight is decreased according to the negative part of the STDP curve. Thus, what matters is not the timing of spiking per se, but the exact timing of arrival of presynaptic spikes to postsynaptic targets.

Rhythms. Initially, all synaptic connections have equal weights, and the network is allowed to settle down for 24 hours of model time (which takes 6 hours on $1 \mathrm{MHz} \mathrm{PC}$ ) so that some synapses are potentiated and others are depressed. At the beginning of this settling period, the network exhibits high-amplitude 

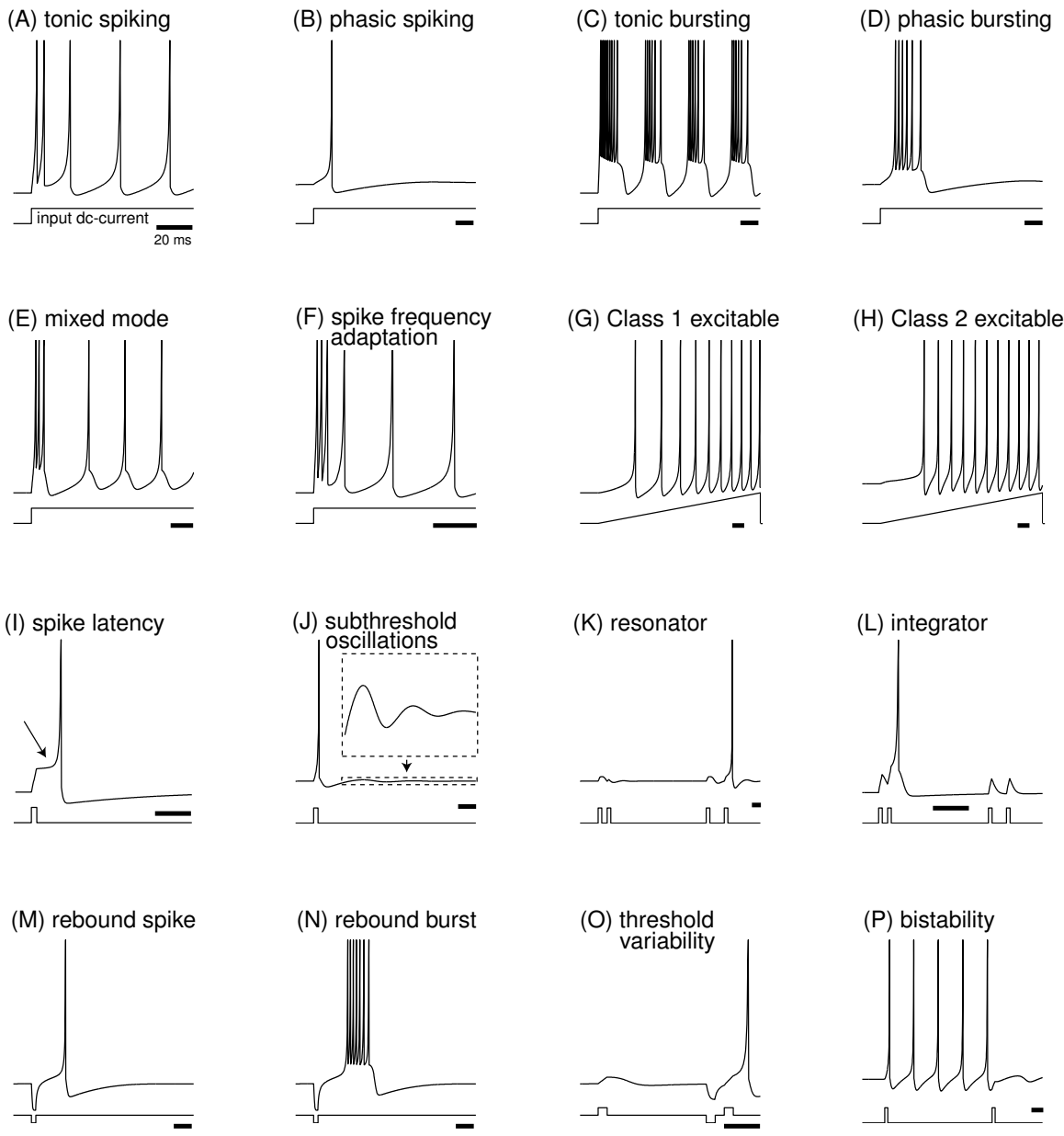

(Q) depolarizing after-potential

(R) accommodation
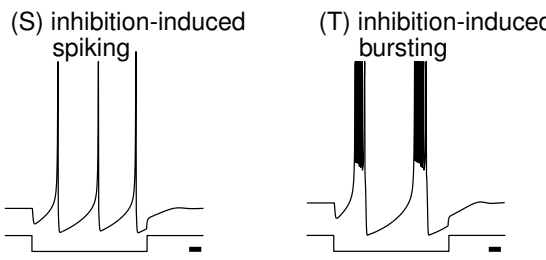

Figure 2: Summary of the neuro-computational properties of biological spiking neurons. Shown are simulations of the same model, Eq. (1, 2), with different choices of parameters. Each horizontal bar denotes $20 \mathrm{~ms}$ time interval. The MATLAB file generating the figure and containing all the parameters can be downloaded from the author's website. This figure is reproduced with permission from www.izhikevich.com. (Electronic version of the figure and reproduction permissions are freely available at www.izhikevich.com). 


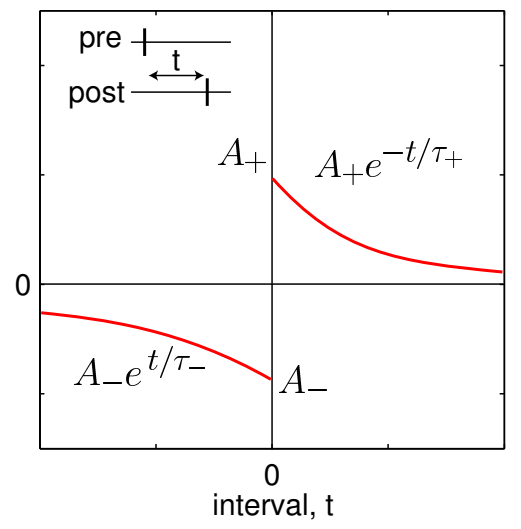

Figure 3: STDP rule: The weight of synaptic connection from pre to postsynaptic neuron is increased if post neuron fired after the pre-synaptic spike, i.e., the interspike interval $t>0$. The magnitude of change decreases as $A_{+} e^{-t / \tau_{+}}$. Reverse order results in decrease of the synaptic weight with magnitude $A_{-} e^{t / \tau_{-}}$. Parameters used: $\tau_{+}=\tau_{-}=20 \mathrm{~ms}, A_{+}=0.1$ and $A_{-}=0.12$.

rhythmic activity in the delta frequency range around $4 \mathrm{~Hz}$, see Fig. 4, top. This is one of the four fundamental types of brain waves, sometimes called "deep sleep waves", because it occurs during dreamless states of sleep, infancy, and in some brain disorders.

As the synaptic connections evolve according to STDP, the delta oscillations disappear, and spiking activity of the neurons becomes more Poissonian and uncorrelated. After a while, gamma frequency rhythms in the range $30-70 \mathrm{~Hz}$ appear, as one can see in Fig. 4, bottom. This kind of oscillations, implicated in cognitive tasks in humans and other animals, play an important role in the activation of polychronous groups, as we will describe in the next section.

Balance of Excitation and Inhibition. There are fewer inhibitory neurons in the network, but their firing rate is proportionally higher, as one can see in Fig. 4. As a result, the network converges to a state with an approximate balance of excitation and inhibition (Shadlen and Newsome 1994, 1999, van Vreeswijk and Sompolinsky 1996, Amit and Brunel 1997), so that each excitatory neuron fires in the Poissonian manner with the rate fluctuating between 2 and $7 \mathrm{~Hz}$. Even during the episodes of gamma oscillation, such as the one in Fig. 4, spiking activity of excitatory neurons is not synchronized; the neurons skip most of the gamma cycles and fire just a few spikes; see dots in Fig. 5, middle.

Two-fold changes of some of the parameters, such as the maximal synaptic weight, the amount of depression in STDP, or the thalamic input, produce only transient changes in network dynamics. Neurons adjust their synaptic weights, balance the excitation and inhibition, and return to the mean firing rate between 2 and $7 \mathrm{~Hz}$. Thus, the network maintains a certain homeostatic state despite 

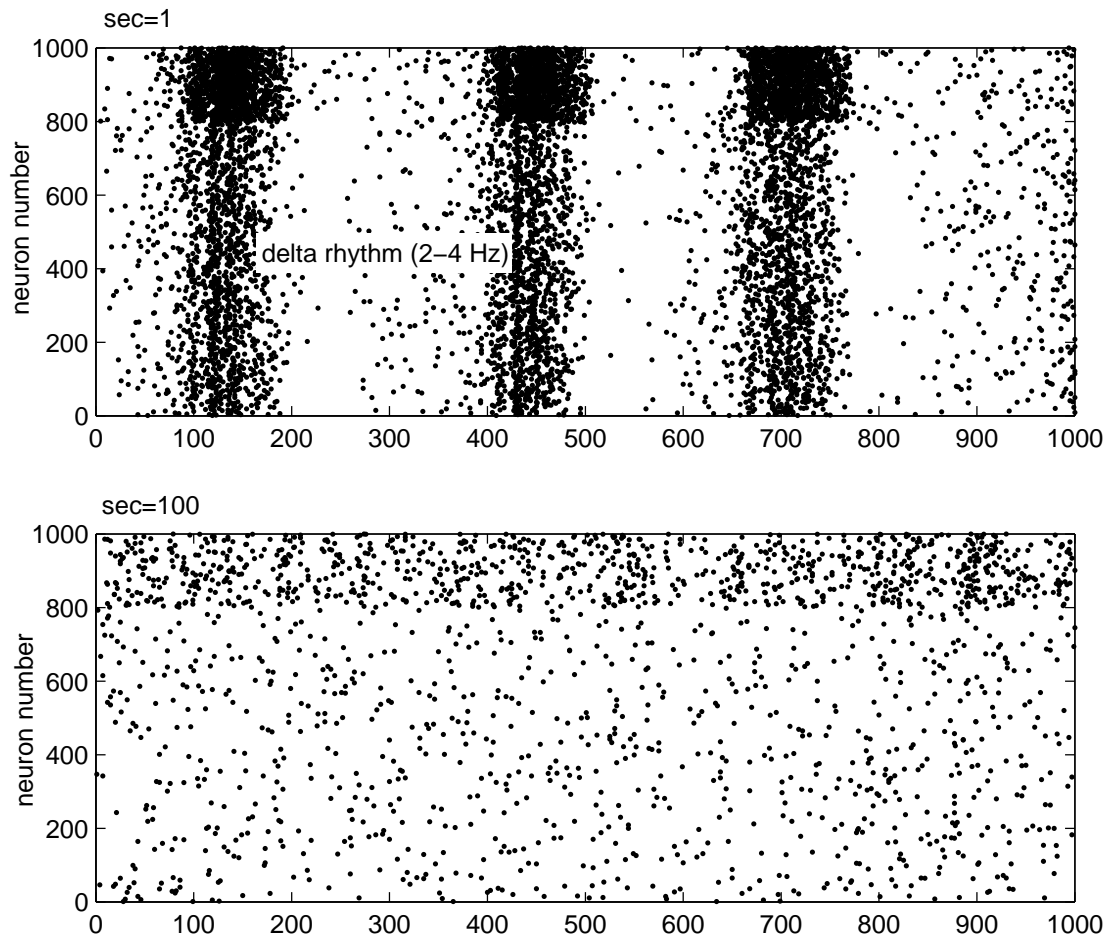

$\mathrm{sec}=3600$

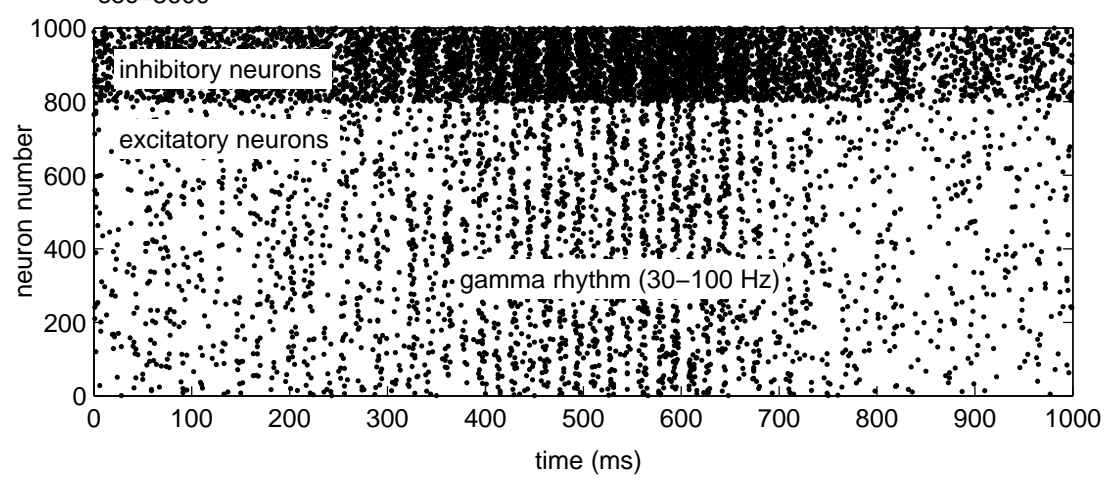

Figure 4: Rhythmic activity of the spiking model. Initial delta frequency oscillations are transformed into gamma frequency oscillations as synaptic weights are evolved according to STDP. 


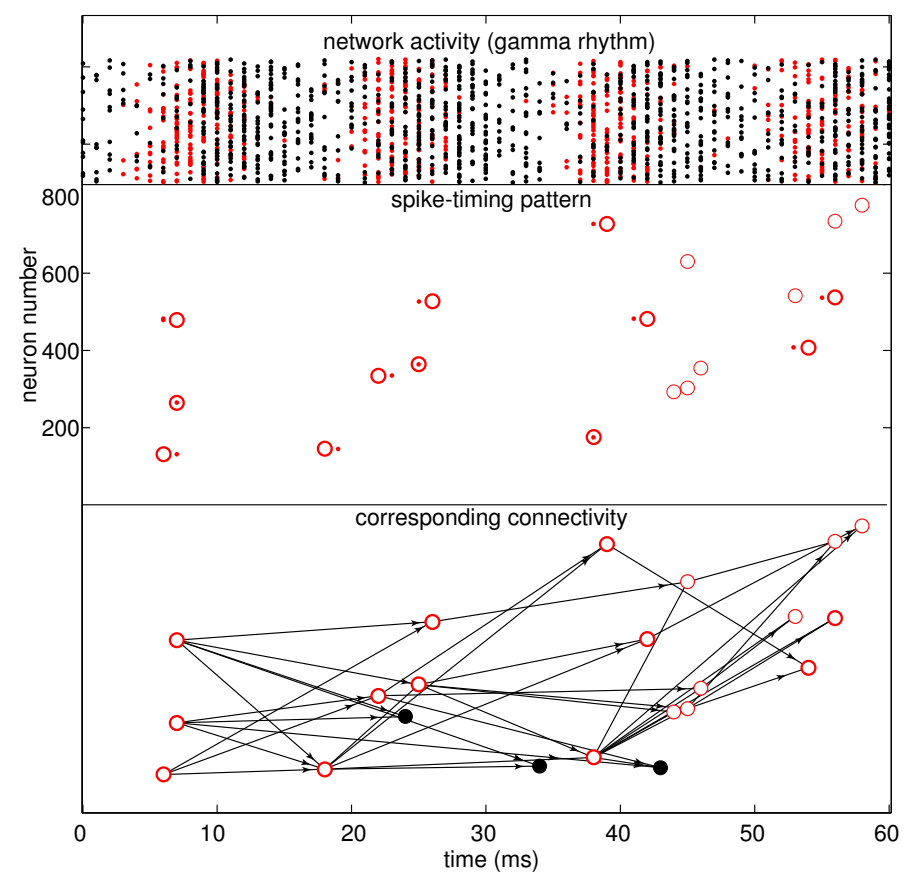

Figure 5: Activation of a polychronous group: Spiking activity of the entire network (top) contains a pattern (middle) that is generated by the neurons comprising a group (bottom). Dots denote actual spikes, circles denote the predicted timing of spikes based on the anatomical connectivity and the delays among neurons.

intrinsic or extrinsic perturbations.

\section{Polychronous Spiking}

Although spiking of excitatory neurons look random and uncorrelated, there are certain persistent spike-timing patterns that emerge and re-occur with a millisecond precision. An example of such a spike-timing pattern is presented in Fig. 5. Though no apparent order can be seen in the network activity at the top of the figure, except for a pronounced gamma oscillation, the pattern denoted by circles in the middle of the figure repeats itself a few times per hour with $\pm 1 \mathrm{~ms}$ spike jitter. Statistical considerations not presented here suggest that such repetitions are highly unlikely to occur by chance. There must be some underling connectivity that generates the pattern. Indeed, considering the connections between the neurons, depicted in Fig. 5, bottom, we can see that the neurons are organized into a group, referred here as being polychronous, i.e., multiple-timing, which forces the neurons to fire with the spiking pattern. 


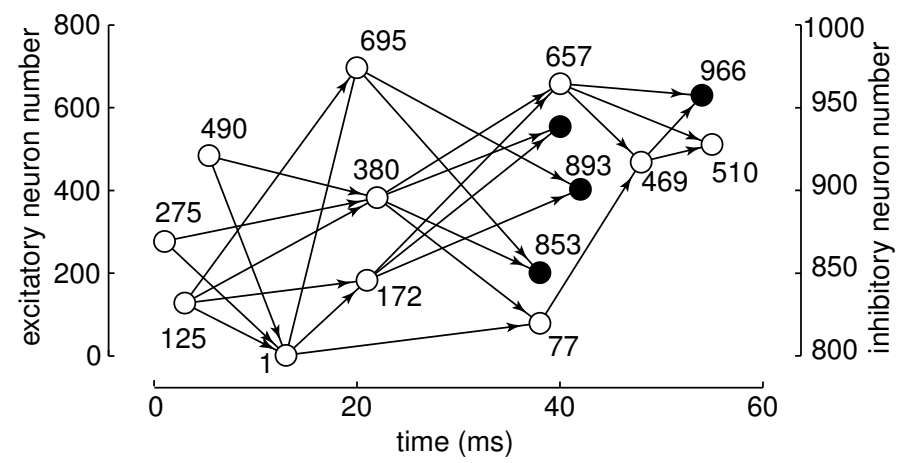

Figure 6: Definition of a polychronous group: Firing of neurons $(125,275,490)$ with the timing pattern $(0,3,7)$ ms results in spikes arriving simultaneously to neuron 1, then to neuron 172, 695, and 380. This multi-timing (polychronous) activity propagates further along the network and terminates at neuron 510 .

Definition. To define polychronous groups precisely, let us consider Fig. 6 . The conduction delays from neurons $(125,275,490)$ to neuron 1 in the figure are such that when the neurons fire with the timing pattern $(0,3,7) \mathrm{ms}$, their spikes arrive at neuron 1 at the same time, thereby making neuron 1 fire at 13 ms. The conduction delays from neurons 125 and 1 to neuron 172 are such that when the neurons fire with the pattern $(3,13) \mathrm{ms}$, their spikes arrive at neuron 172 simultaneously, thereby making it fire at $21 \mathrm{~ms}$. Since we know all delays in the model, we can continue this procedure spike by spike and untangle the entire group. It consists of 15 neurons, some of them are inhibitory, and the group ends at neuron 510 .

Whenever the neurons in the figure fire with the spike-timing pattern determined by the connectivity and delays, we say that the group is activated. Often, firing of the first few neurons with the right timing is enough to activate most of the group, as it happens in Fig. 5. Notice how activation of the group is locked to the gamma oscillation; that is, the first three neurons fire at the first gamma cycle, their spikes travel 10-20 ms and arrive to the next four neurons in the next gamma cycle, and so on.

Examples. Considering various triplets, such as neurons $(125,275,490)$ in Fig. 6, left, firing with various patterns, we can reveal all polychronous groups. In the network of 1000 neurons presented in Appendix, we find 5269 such groups. They have different sizes, lengths, and time spans, as we summarize in Fig. 7. A few examples are depicted in Fig. 10. Since an averaged group consists of 25 neurons, an averaged neuron is a member of 131 different groups. Because different groups activate at different times, the neuron can fire with one group at one time and with another group at another time.

Quite often different polychronous groups can share more than one neuron. Two such cases are illustrated in Fig. 8. Neurons $(8,103,351,609,711,883)$ 

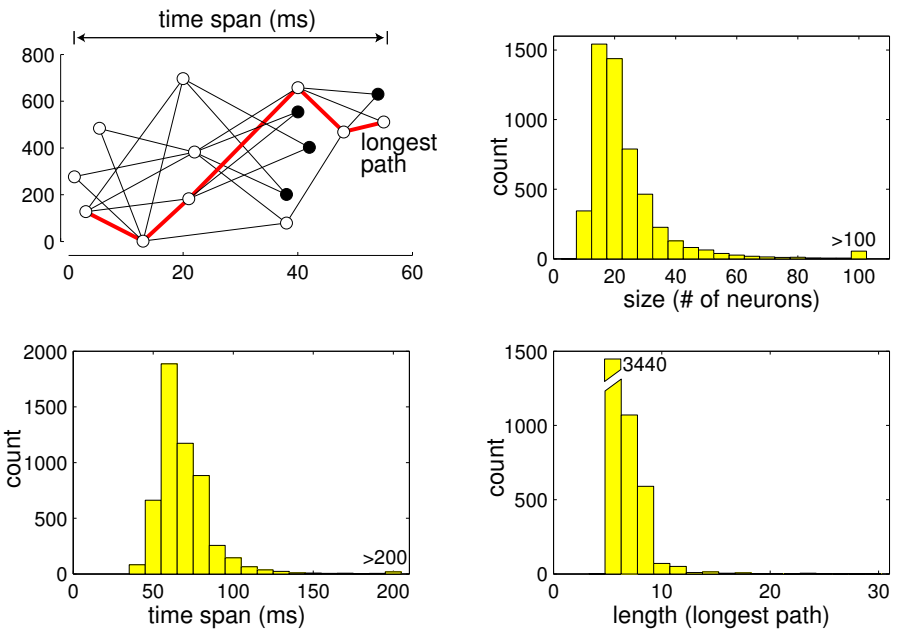

Figure 7: Characteristics of polychronous groups (total number is 5269 in a network of 1000 neurons). Each group is characterized by the number of neurons that form the group, its time span and its longest path.

belong to two groups in the upper half of the figure. However, there is no ambiguity because their firing order is different; the neurons fire with one spiketiming pattern at one time (when the first group is activated) and with the other pattern at some other time (when the second group is activated). The lower half of the figure depicts two groups having 8 neurons in common, and firing with different spike-timing patterns. In addition, neurons 838 and 983 fire twice during activation of the second group. Again, there is no ambiguity here because each polychronous group is defined not only by its constituent neurons, but also by their precise spiking time. In principle, there could be two groups consisting of exactly the same neurons but firing with completely different spike-timing patterns, though we did not find such groups in the model.

\section{Cognitive Computations}

Let us discuss how polychronous groups can contribute to cognitive computations of the network.

Rate to Spike-Timing Conversion. Neurons in the model use spike-timing code to interact and form groups. However, the external input from sensory organs, such retinal cells, hair cells in cochlear, etc., arrives as the rate code, i.e., encoded into the mean firing frequency of spiking. How can the network convert rates to precise spike timings?

In Fig. 9 we describe the mechanism of such conversion, which is similar, but not equivalent, to the mechanism proposed by Hopfield (1995). A volley of 

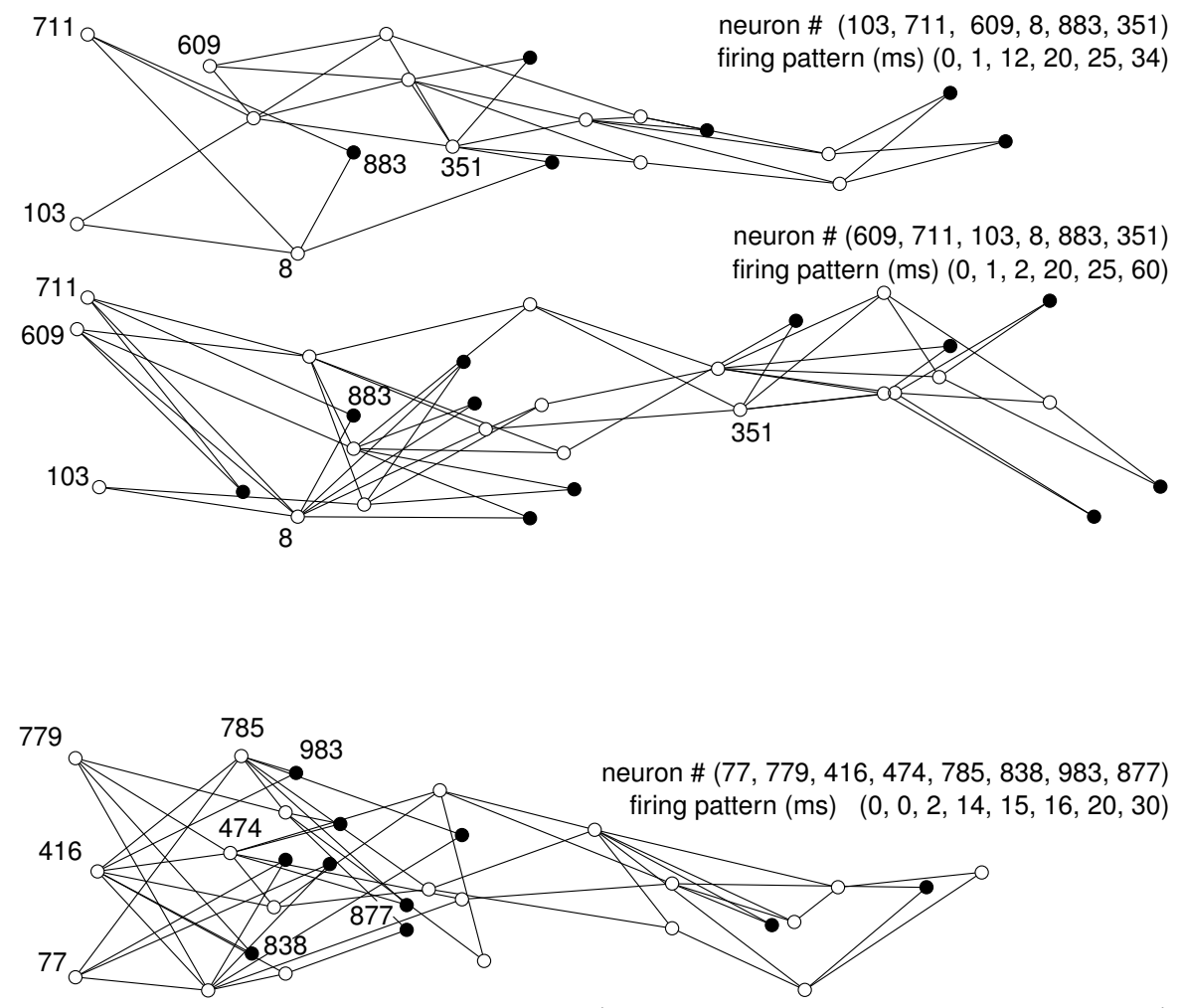

neuron \# $(77,416,779,785,474,838,983,877,838,983)$
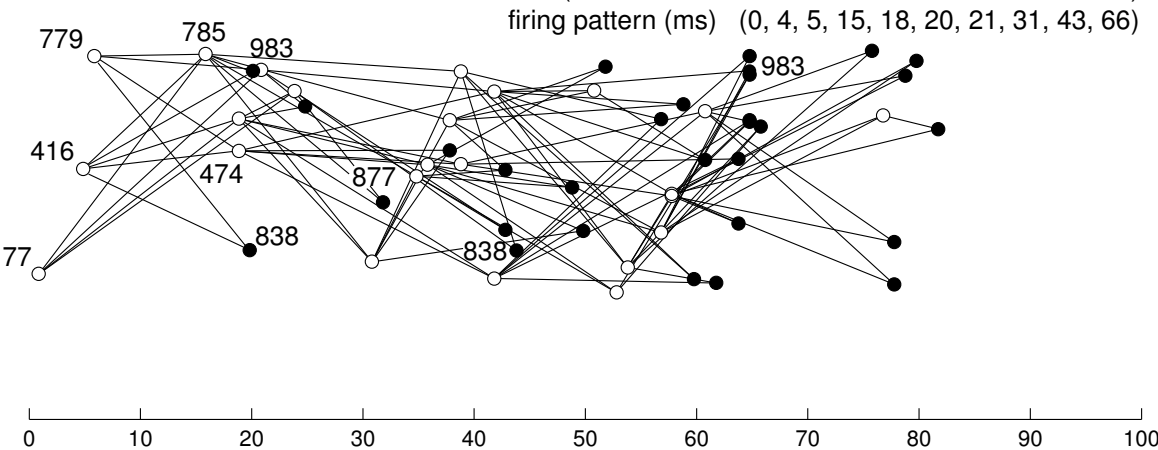

time (ms)

Figure 8: Different firing patterns of the same neurons can result in different polychronous groups. 


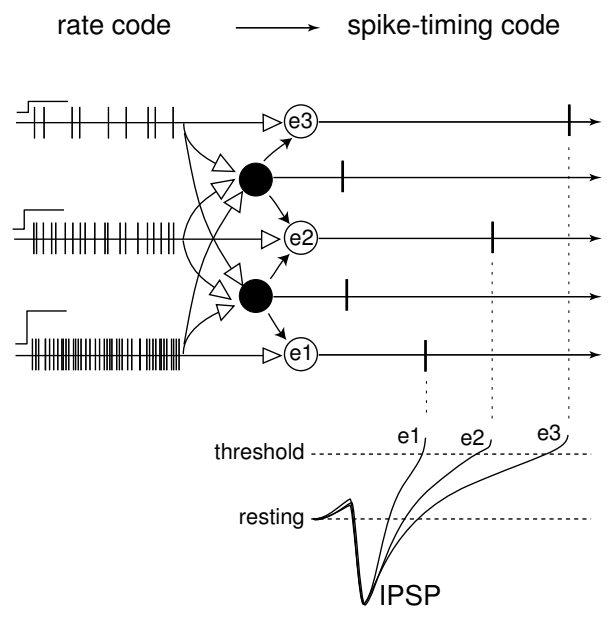

Figure 9: Rate code to spike-timing code conversion by spiking network with fast inhibition. Open circles - excitatory neurons, black circles - inhibitory neurons..

external input excites inhibitory and excitatory neurons. The inhibitory neurons, being faster, fire first and inhibit excitatory neurons thereby resulting in a long inhibitory postsynaptic potential (IPSP). The rate with which excitatory neurons recover from the IPSP depends on their intrinsic properties and on the strength of the overall external input. The stronger the input, the sooner the neuron fires after the IPSP. We see that the neuron receiving the strongest input fired first, and the neuron receiving the weakest input fired last.

Since the recovery from inhibition is nearly exponential, the system is relatively insensitive to the input scaling. That is, a stronger input results in firing with the same spike-timing pattern, but with an earlier onset. Similarly, a weaker input does not change the spike-timing pattern, but only delays its onset. Thus, in contrast to Hopfield (1995), we do not need to postulate that the input is already somehow converted to logarithmic scale. Synchronized inhibitory spiking implements the logarithmic conversion that makes spike-timing relatively insensitive to the input scaling.

Notice that synchronized inhibitory activity occurs during gamma frequency oscillations. Thus, the network constantly converts rate code to spike-timing code (and back) via gamma rhythm. The functional implications of such a non-stop conversion are not clear.

Representations. What is the significance of polychronous groups? We hypothesize that polychronous groups could represent memories and experience. In the simulation above, no coherent external input to the system was present. As a result, random groups emerge; that is, the network generates random memories not related to any previous experience.

However, coherent external stimulation builds certain groups that represent 


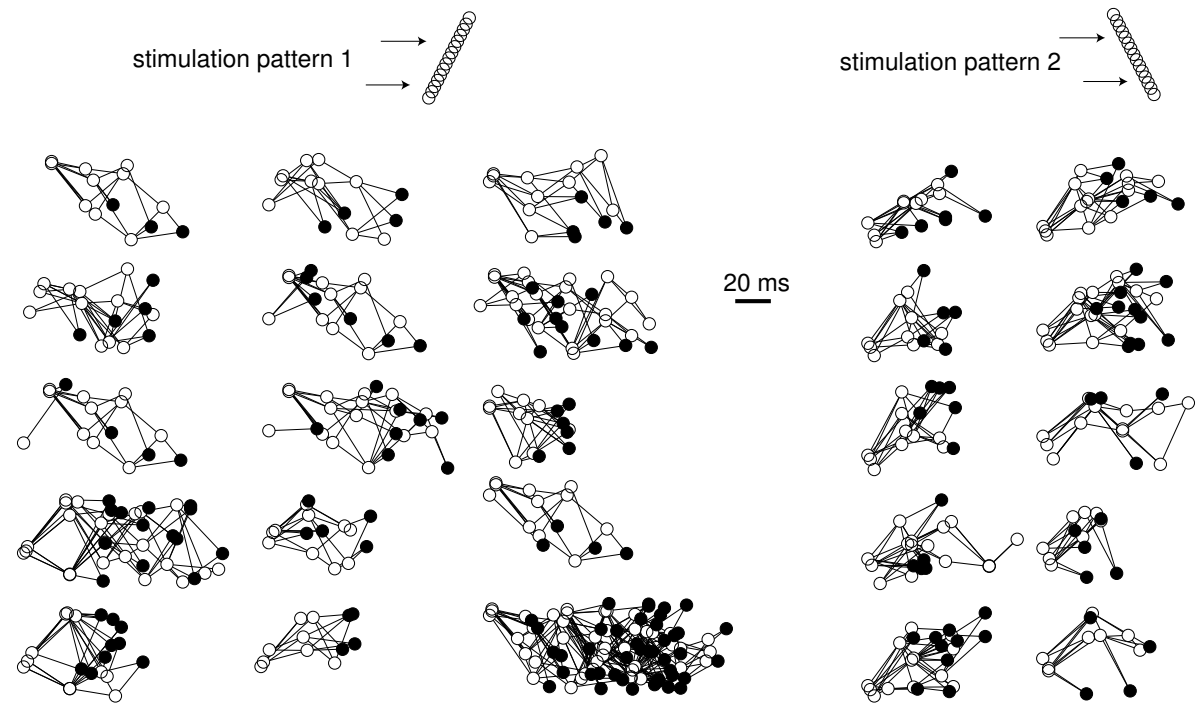

Figure 10: Persistent stimulation of the network with two spatio-temporal patterns result in emergence of polychronous groups that represent the patterns; i.e., the groups activate whenever the patterns are present.

this stimulation in the sense that the groups are activated when the stimulation is present. Different stimuli build different groups even when the same neurons are stimulated, as we illustrate in Fig. 10. Every second during a 20-minute period, we stimulate 40 neurons, $1,21,41,61, \ldots 781$, either with the pattern $(1,2, \ldots, 40) \mathrm{ms}$ or with the pattern $(40, \ldots 2,1) \mathrm{ms}$, as we show in the top of Fig. 10. Initially, no groups corresponding to these stimuli existed, however after 20 minutes of simulation 25 new groups emerged. 15 of them correspond to the first stimulus, i.e., they can be activated when the network is stimulated with the first pattern. The other 10 correspond to the second stimulus; that is, they can be activated when the network is stimulated with the second pattern. Thus, the groups represent the memory of the two input patterns, and their activation occurs when the network "sees" the corresponding patterns.

In Fig. 11 we depict the time evolution of the largest group corresponding to the first pattern in Fig. 10. Notice how the group annexes neurons, probably at the expense of the other groups in the network. Further simulation shows that the initial portion of the group is relatively stable, but its tail expands and shrinks in an unpredictable manner.

Finally, not all groups corresponding to a pattern activate when the network is stimulated. Because the groups share some neurons and have excitatory and inhibitory interconnections, they are in the constant state of competition and cooperation. As a result, each presentation of a stimulus activates only 2-3 groups in a random manner. 


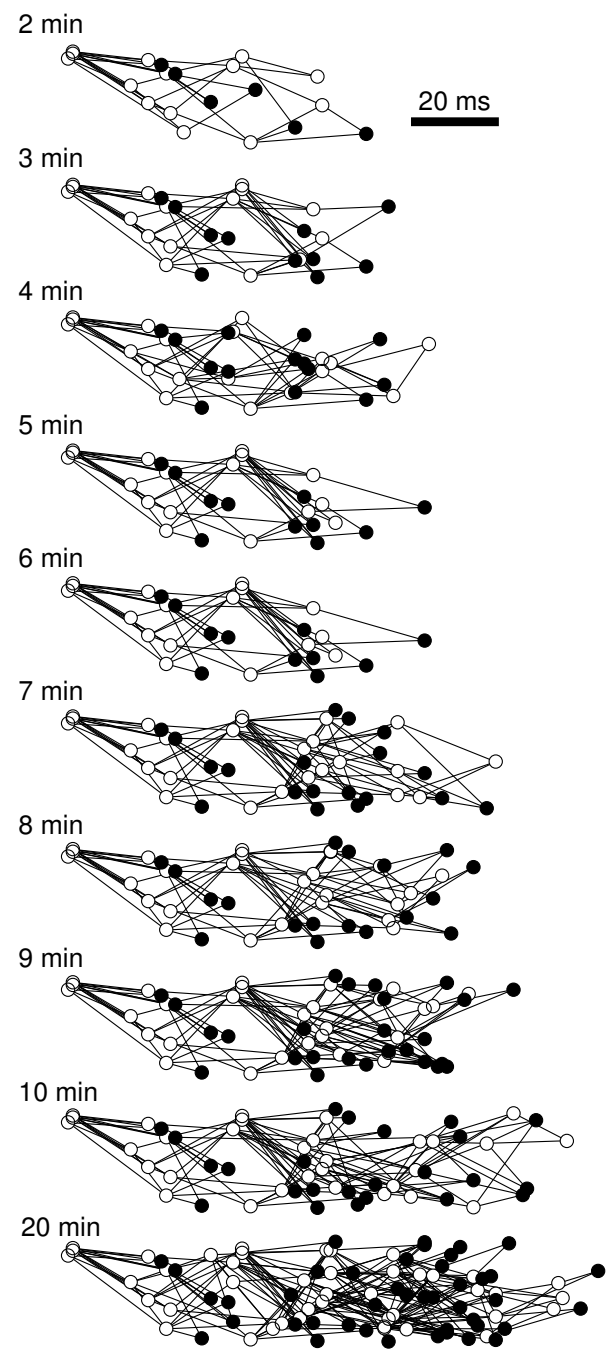

Figure 11: Evolution of the last (largest) polychronous group in Fig. 10 corresponding to stimulation pattern 1. 


\section{Discussion}

Simulating a simple network of spiking neurons with axonal conduction delays and spike-timing-dependent plasticity (STDP), we discovered a number of interesting phenomena. The most striking one is the emergence of polychronous groups, i.e., strongly interconnected groups of neurons having matching conduction delays and capable of firing time-locked spikes with millisecond precision. Thus, such groups can be seen not only in anatomically detailed cortical models (Izhikevich 2004), but also in simple spiking networks. A ready-to-use MATLAB file implementing the network is presented in the appendix.

How is it different from synfire chains? The notion of polychronous groups is quite different from the notion of synfire chains (Abeles 1991, Bienenstock 1995, Diesmann et al. 1999, Ikegaya et al. 2004), which describes pools of neurons firing synchronously. Synfire activity relies on synaptic connections having equal delays or no delays at all. Though easy to implement, networks without delays are finite-dimensional and do not have rich dynamics to support persistent polychronous spiking. Indeed, in the context of synfire activity, the groups in Fig. 8 could not be distinguished. Bienenstock (1995) referred to such an activity as a synfire braid.

Synfire chain research concerns the stability of a synfire activity. Instead, we employ here the population thinking (Edelman 1987): Even though many polychronous groups are short-lived, there is a huge number of them constantly appearing. Even though their activation is not reliable, tens of them activate every second in a network of 1000 neurons. Thus, the system is robust not in terms of individual groups, but in terms of populations of groups.

How is it different from Hopfield-Grossberg attractor networks? Polychronous groups are not attractors from dynamical system point of view. When activated, they result in stereotypical but transient activity that typically lasts 3-4 gamma cycles (less than $100 \mathrm{~ms}$ ); see Fig. 7. Once the stimulation is removed, the network does not return to a "default" state, but continues to be active.

How is it different from feed-forward networks? The anatomy of the spiking networks that we consider is not feed-forward, but reentrant (Edelman 1987). Thus, the network does not "wait" for stimulus to come, but exhibits an autonomous activity. Stimulation only perturbs the intrinsic activity, as it happens in mammalian brain. As a result, the network does not have a rigid stimulus-response function. The same stimulus can elicit quite different responses because it activates a different (random) subset of polychronous groups representing the stimulus. Thus, the network operates in a highly degenerate regime (Edelman and Gally 2001). 
How is it different from Liquid-State-Machines? Dynamics of a network implementing the liquid-state-machine paradigm (Maass 2002) is purely stimulus driven. Such a network does not have short-term memory and it cannot place the input in the context of the previous inputs. The simple model presented here implements some aspects of the liquid-state computing, e.g., it could be the liquid, however its response is not quite stimulus-driven; It depends on the current state of the network which in turn depends on the short-term and long-term experience and previous stimuli. This could be an advantage or a drawback, depending on the task that needs to be solved.

Open Problems. Let us mention some open problems and issues.

- (Finding Groups) Our algorithm of finding polychronous groups considers various triplets firing with various spiking patterns and determines the groups that are initiated by the patterns. Because of the combinatorial explosion, it is extremely inefficient. In addition, we probably miss many groups that do not start with three neurons.

- (Training) Our training strategy is the simplest and probably the least effective one: Choose a set of "sensory" neurons, stimulate them with different spike-timing sequences, and let STDP form the corresponding groups. It is not clear whether this strategy is effective when many stimuli are needed to be learned.

- (Incomplete Activation) When a group is activated, whether in response to a particular stimulation or spontaneously, it rarely activates entirely. Typically, neurons at the beginning of the group fire with the precise spike-timing pattern imposed by the group connectivity, but the precision fades away as activation propagates along the group. As a result, the connectivity in the tail of the group does not stabilize, so the group as a whole changes.

- (Stability) Because of continuous plasticity, groups appear, grow (see Fig. 11), live for certain period of time, and then could suddenly disappear (Izhikevich 2004). Thus, spontaneous activity of the network leads to a slow degradation of the memory, and it is not clear how to prevent this.

- (Sleep States) The network can switch randomly between different states, some of them correspond to "vigilance" with gamma oscillations, others correspond to "sleep" states, similar to the one in Fig. 4, top. It is not clear whether such switching should be prevented or whether it provides certain advantages for homeostasis of connections.

- (Optimizing Performance) Exploring the model, we encounter a regime when the number of polychronous groups was greater than the number of synapses in the network. However, the network was prone to epileptic seizures, which eventually lead to uncontrolled completely synchronized activity. More effort should be done to fine-tune the parameters of 
the model to optimize the performance of the network without inducing paroxysmal behavior.

- (Context Dependence) Propagation delays are assumed to be constant in the present simulation. In vivo studies have shown that axonal conduction velocity depends on the prior activity of the neuron, hence it can change with time in a context-dependent manner. Thus, a polychronous group may exist and be activated in one time, but can temporary disappear at another time because of the previous activity of its constituent neurons.

- (Scaling) We simulated a network of $10^{3}$ neurons and found $10^{4}$ polychronous groups. How does the number of groups scales with the number of neurons? In particular, how many polychronous groups are there in a network of $10^{11}$ neurons? This is a fundamental question related to the information capacity of the human brain.

\section{Further Directions}

Closing the Loop. An important biological observation is that organisms are part of the environment. Thus, to understanding and simulate the brain, we need to give the neural network a body and put it into real or virtual environment (Krichmar 2002). In this case, the network becomes part of a closed loop: The environment stimulates "sensory" neurons via sensory organs. Firings of these neurons combined with the current state of the network (i.e. the context) activate appropriate polychronous groups, which excite "motor" neurons and produce appropriate movements in the environment (i.e., response). The movements change the sensory input and close the causality loop.

Some stimuli bring the reward and activate the value system (Krichmar 2002). It strengthens recently active polychronous groups, i.e., the groups that resulted in the reward. Thus, in addition to passively learning input stimuli, the system can actively explore those stimuli that bring the reward.

Modeling Attention. The small size of the system does not allow us to explore other cognitive functions of spiking networks. In a large-scale network, there could be many groups (more than 15 depicted in Fig. 10) that represent any particular input stimulus. Whenever the network 'sees" the stimulus, a random subset of those groups is activated, corresponding to perception of the stimulus. This subset may take up all the neurons in the network so that no neurons are available for activation of any other group not related to the stimulus. If two or more stimuli are present, then activation of groups representing one stimulus essentially excludes the other stimuli from being perceived. This winner-take-all sequential perception may be related to the phenomenon of selective attention.

Consciousness. When no stimulation is present, there is a spontaneous activation of polychronous groups. We hypothesize that if the size of the network 
exceeds certain threshold, a random activation of a few groups corresponding to a previously seen stimulus may activate other groups corresponding to the same stimulus so that the total number of activated groups is comparable to the number of activated groups that occurs when the stimulus is present. Not only such an event excludes all the other groups not related to the stimulus from being activated, but from the network point of view, it would be indistinguishable from the event when the stimulus is actually present. One can say that the network "thinks" about the stimulus. A sequence of spontaneous activations corresponding to one stimulus, then another, and so on, may be related to the stream of thought and primary consciousness (Edelman 2004).

\section{A The Model}

The MATLAB code simulating the network activity is in Fig. 12. The upper half of the program initializes the network, and it takes approximately $30 \mathrm{sec}$ on $1 \mathrm{GHz}$ Pentium PC. The lower half of the program executes the model, and it takes 5 seconds to simulate 1 second of network activity. The actual time may vary depending on the firing rate of the neurons.

The MATLAB code and an equivalent 20-times faster $\mathrm{C}++$ code are also available on the author's webpage. Let us describe the details of the model.

Anatomy. The network consists of $\mathrm{N}=1000$ neurons with the first $\mathrm{Ne}=800$ of excitatory RS type, and the remaining $\mathrm{Ni}=200$ of inhibitory FS type (Izhikevich 2003). The ratio of excitatory to inhibitory cells is 4 to 1 , as in the mammalian neocortex. Each excitatory neuron is connected to $M=100$ random neurons, so that the probability of connection is $\mathrm{M} / \mathrm{N}=0.1$, again as in the neocortex. Each inhibitory neuron is connected to $\mathrm{M}=100$ excitatory neurons only. The indices of postsynaptic targets are in the $\mathrm{N} \times \mathrm{M}$-matrix post. Corresponding synaptic weights are in the $\mathrm{N} \times \mathrm{M}$-matrix $\mathbf{s}$. Inhibitory weights are not plastic, whereas excitatory weights evolve according to the STDP rule discussed in the next section. Their derivatives are in the $\mathrm{N} \times \mathrm{M}$-matrix sd, though only the $\mathrm{Ne} \times \mathrm{M}$ block of the matrix is used.

Each synaptic connection has a fixed conduction delay between $1 \mathrm{~ms}$ and $\mathrm{D}=20 \mathrm{~ms}$, where $\mathrm{D}$ is a parameter (M/D must be integer in the model). The list of all synaptic connections from neuron $\mathrm{i}$ having delay $\mathrm{j}$ is in the cell array delays $\{i, j\}$. Our MATLAB implementation assigns $1 \mathrm{~ms}$ delay to all inhibitory connections, and 1 to D ms delay to all excitatory connections. Even though the anatomy of the model is random reflecting the connectivity within a cortical minicolumn, one can implement an arbitrary sophisticated anatomy by specifying the matrices post and delays. The details of the anatomy do not matter in the rest of the MATLAB code, nor do they slow down the simulation.

Once the matrices post and delays are specified, the program initializes cell arrays pre and aux. The former contains indices of all excitatory neurons presynaptic to a given neuron, and the latter is an auxiliary table of indices needed to speed up STDP implementation. 


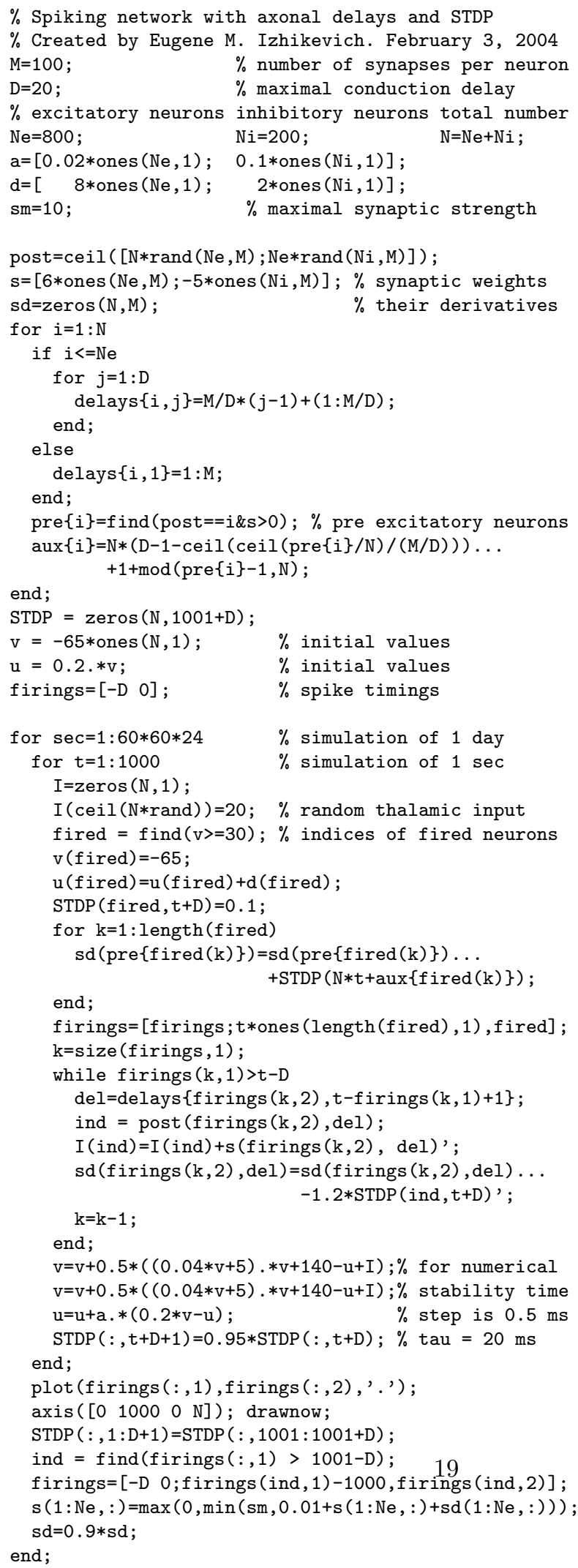

Figure 12: MATLAB code of the spiking network with axonal conduction delays and spike-timing-dependent plasticity (STDP). 
Spiking Neurons. Each neuron in the network is described by the simple spiking model (Izhikevich 2003)

$$
\begin{aligned}
v^{\prime} & =0.04 v^{2}+5 v+140-u+I \\
u^{\prime} & =a(b v-u)
\end{aligned}
$$

with the auxiliary after-spike resetting

$$
\text { if } v \geq+30 \mathrm{mV} \text {, then }\left\{\begin{array}{l}
v \leftarrow c \\
u \leftarrow u+d .
\end{array}\right.
$$

Here variable $v$ represents the membrane potential of the neuron and $u$ represents a membrane recovery variable, which accounts for the activation of $\mathrm{K}^{+}$ ionic currents and inactivation of $\mathrm{Na}^{+}$ionic currents, and it provides negative feedback to $v$. After the spike reaches its apex at $+30 \mathrm{mV}$, which is not to be confused with the firing threshold, the membrane voltage and the recovery variable are reset according to the equation (3). Depending on the values of the parameters, the model can exhibit firing patterns of all known types of cortical neurons (Izhikevich 2003). It can also reproduce all of the 20 most fundamental neuro-computational properties of biological neurons summarized in Fig. 2; see (Izhikevich 2004).

We use $(b, c)=(0.2,-65)$ for all neurons in the network. For excitatory neurons we use the values $(a, d)=(0.02,8)$ corresponding to cortical pyramidal neurons exhibiting regular spiking (RS) firing patterns. For inhibitory neurons we use the values $(a, d)=(0.1,2)$ corresponding to cortical inter-neurons exhibiting fast spiking (FS) firing patterns.

Variable $I$ in the model combines two kinds of input to the neuron: (1) random thalamic input, and (2) spiking input from the other neurons. This is implemented via $\mathrm{N}$-dimensional vector $\mathrm{I}$.

Spike-Timing-Dependent Plasticity. The synaptic connections in the model are modified according to the spike-timing-dependent plasticity (STDP) rule (Song et al. 2000). We use the simplest and the most effective implementation of this rule, depicted in Fig. 3. If a spike from an excitatory presynaptic neuron arrives to a postsynaptic neuron and makes the postsynaptic neuron fire, then the synapse is potentiated (strengthened). In contrast, if the spike arrives right after the postsynaptic neuron fired, the synapse is depressed (weakened).

If pre- and postsynaptic neurons fire uncorrelated Poissonian spike trains, then there are moments when the weight of the synaptic connection is potentiated and there are moments when it is depressed. We chose the parameters of the STDP curve so that depression is stronger than potentiation and the synaptic weight goes slowly to zero. Indeed, such a connection is not needed and should be eliminated. In contrast, if the presynaptic neuron often fires before the postsynaptic one, then the synaptic connection slowly potentiates. Indeed, such a connection causes the postsynaptic spikes and should be strengthened. In this way, STDP strengths causal interactions in the network. 
The magnitude of potentiation or depression depends on the time interval between the spikes. Each time a neuron fires, the variable STDP is reset to 0.1. Every millisecond, STDP decreases by $0.95 *$ STDP, so that it decays to zero as

$0.1 e^{-t / 20(\mathrm{~ms})}$, according to the parameters in Fig. 3. This function determines the magnitude of potentiation or depression.

For each fired neuron we consider all its presynaptic neurons and determine the timings of last excitatory spikes arrived from these neurons. Since these spikes made the neuron fire, the synaptic weights are potentiated according to the value of STDP at the presynaptic neuron adjusted for the conduction delay. This corresponds to the positive part of the STDP curve in Fig. 3. Notice that the largest increase occurs for the spikes that arrived right before the neuron fired, that is, for the spikes that actually caused postsynaptic spike.

In addition, when an excitatory spike arrives to a postsynaptic neuron, we depress the synapse according to the value of STDP at the postsynaptic neuron. This corresponds to the negative part of the STDP curve in Fig. 3. Indeed, such a spike arrived after the postsynaptic neuron fired, and hence the synapse between the neurons should be weakened. (The same synapse will be potentiated when the postsynaptic neuron fires.)

Instead of changing the synaptic weights directly, we change their derivatives sd, and then update the weights once a second according to the rule $\mathbf{s} \leftarrow \mathbf{s}+0.01$ $+\mathrm{sd}$, and $\mathrm{sd} \leftarrow 0.9 \mathrm{sd}$, where 0.01 describes activity-independent increase of synaptic weight needed to potentiate synapses coming to silent neurons. Thus, the synaptic change is not instantaneous, but slow taking many seconds to develop. We manually keep the weights in the range between 0 and sm, where $\mathrm{sm}$ is a parameter of the model, typically less than $10(\mathrm{mV})$.

\section{Acknowledgment}

Anil K. Seth read the first draft of the manuscript and made a number of useful suggestions. This research was supported by the Neurosciences Research Foundation.

\section{References}

[1] Abeles M. (1991) Corticonics: Neural Circuits of the Cerebral Cortex. Cambridge University Press.

[2] Amit D. J. and Brunel N. (1997) Model of global spontaneous activity and local structured activity during delay periods in the cerebral cortex. Cereb Cortex. 7:237-52

[3] Bienenstock E. (1995) A model of neocortex. Network: Comput. Neural Syst. 6:179-224 
[4] Braitenberg and Schuz (1991) Anatomy of the cortex : statistics and geometry, Springer Verlag.

[5] Buonomano D.V. (2003) Timing of neuronal responses in cortical organotypic slices. PNAS, 100:4897-4902

[6] Diesmann M., Gewaltig M.-O., Aertsen A. (1999) Stable propagation of synchronous spiking in cortical neural networks. Nature402, $529-533$.

[7] Edelman G.M. (1987) Neural Darwinism: The Theory of Neuronal Group Selection. Basic Books.

[8] Edelman G.M. (2004) Wider than the Sky: The Phenomenal Gift of Consciousness. New Haven: Yale University Press.

[9] Edelman G.M. and Gally J. (2001) Degeneracy and complexity in biological systems. PNAS, 98:13763-13768

[10] Gerstner W., Kempter R., van Hemmen J. L., Wagner H. (1996) A neuronal learning rule for sub-millisecond temporal coding Nature, 383: 76 - 78

[11] Grutzendler J., Kasthuri N., and Gan W.-B. (2002) Long-term dendritic spine stability in the adult cortex. Nature, 420:812-816.

[12] Hopfield, J. J. (1995) Pattern recognition computation using action potential timing for stimulus representation. Nature 376, 33-6.

[13] Ikegaya Y., Aaron G., Cossart R., Aronov D., Lampl I., Fester D., Yuste R. (2004) Synfire Chains and Cortical Songs: Temporal Modules of Cortical Activity. Science, 304:559-564.

[14] Izhikevich E.M. (2004) Which Model to Use for Cortical Spiking Neurons? IEEE Transactions on Neural Networks, 15:1063-1070

[15] Izhikevich E.M. , Gally J.A., and Edelman G.M. (2004) Spike-Timing Dynamics of Neuronal Groups. Cerebral Cortex, accepted.

[16] Izhikevich E.M. (2003) Simple Model of Spiking Neurons, IEEE Transactions on Neural Networks, 14:1569-1572.

[17] Krichmar J.L. , Edelman G.M., (2002) Machine Psychology: Autonomous Behavior, Perceptual Categorization and Conditioning in a Brain-Based Device, Cerebral Cortex 12:818-830

[18] Maass, W., Natschlaeger, T. \& Markram, H. (2002), 'Real-time computing without stable states: A new framework for neural computation based on perturbations', Neural Computation 14, 2531-2560. 
[19] Seth, A. K., Mckinstry, J. L., Edelman, G. M., \& Krichmar, J. L. (2004). Spatiotemporal processing of whisker input supports texture discrimination by a brain-based device. In Schaal. S., Billard, A., Vijayakumar, S., Hallam, J., \& Meyer, J.-A., From Animals to Animats 8: Proceedings of the Eighth International Conference on the Simulation of Adaptive Behavior. Los Angeles, CA: MIT Press.

[20] Shadlen M. N. and Newsome W. T. (1994) Noise, neural codes and cortical organization. Curr. Opin. Neurobiol. 4:569-579

[21] Shadlen, M.N. and Newsome, W.T. (1998) The variable discharge of cortical neurons: implications for connectivity, computation and information coding. J. Neurosci. 18, 3870-3896

[22] Song, S., Miller, K. D., \& Abbott, L. F. (2000). Competitive Hebbian learning through spike-timing-dependent synaptic plasticity. Nature Neurosci., 3 , 919- 926.

[23] Swadlow H. A. (1992) Monitoring the excitability of neocortical efferent neurons to direct activation by extracellular current pulses. J Neurophysiol, 68: $605-619$.

[24] Swadlow H. A. (1988) Efferent Neurons and Suspected Interneurons in Binocular Visual Cortex of the Awake Rabbit: Receptive Fields and Binocular Properties. J Neurophysiol, 88: 1162 - 1187.

[25] Swadlow H. A. (1985) Physiological Properties of Individual Cerebral Axons Studied in Vivo For as Long as One Year. J. of Neurophysiology 54:13461362

[26] Trachtenberg J.T., Chen B.E., Knott G.W., Feng G., Sanes J.R., Welker E., Svoboda K. (2002) Long-term in vivo imaging of experience-dependent synaptic plasticity in adult cortex. Nature 420:788-792.

[27] van Vreeswijk C, Sompolinsky H. (1996) Chaos in neuronal networks with balanced excitatory and inhibitory activity. Science. 274:1724-1726. 